\title{
Direct Evidence of Progressive Cardiac Dysfunction in a Transgenic Mouse Model of Huntington's Disease
}

\author{
Nigel I. Wood ${ }^{\mathrm{a}}$, Stephen J. Sawiak ${ }^{\mathrm{b}, \mathrm{d}}$, Guido Buonincontri ${ }^{\mathrm{b}}$, Youguo Niu ${ }^{\mathrm{c}}$, Andrew D. Kane, \\ T. Adrian Carpenter ${ }^{\mathrm{b}}$, Dino A. Giussani ${ }^{\mathrm{c}}$ and A. Jennifer Morton ${ }^{\mathrm{a}, *}$ \\ ${ }^{a}$ Department of Pharmacology, University of Cambridge, Cambridge, UK \\ ${ }^{\mathrm{b}}$ Wolfson Brain Imaging Centre, University of Cambridge, Addenbrooke's Hospital, Cambridge, UK \\ ${ }^{\mathrm{c}}$ Department of Physiology, Development and Neuroscience, University of Cambridge, Cambridge, UK \\ ${ }^{\mathrm{d}}$ Behavioural and Clinical Neuroscience Institute, Cambridge, UK
}

\begin{abstract}
HD is a progressive genetic neurological disorder, characterized by motor as well as cognitive impairments. The gene carrying the mutation causing Huntington's disease (HD) is not brain specific, and there is increasing evidence for peripheral, as well as brain pathology in this disorder. Here, we used in vivo and ex vivo techniques to assess the cardiac function of mice transgenic for the HD mutation. Using magnetic resonance imaging (MRI) of the beating heart, we show that abnormalities previously reported in end-stage mice are present by mid-stages of the disease. We also found abnormalities that have not been hitherto reported, including changes in cardiac efficiency and a mechanical distortion of the beating heart. Using the Langendorff preparation, we show reduced coronary blood flow, impaired myocardial contractility and reduced left ventricular developed pressure in HD mouse hearts. Together, our findings suggest that there is significant pathology of the HD mouse heart, even by mid stages of disease. Previous clinical research has demonstrated that the risk of cognitive symptoms increases markedly in patients with heart failure. R6/2 mice show significant progressive cognitive abnormalities, so we hypothesize that cardiac pathology in the R6/2 mouse may contribute, not only to their progressive decline and death, but also to their cognitive dysfunction. We suggest that closer attention should be paid to cardiovascular symptoms in HD patients.
\end{abstract}

Keywords: Huntington's disease, R6/2 mouse, cardiac dysfunction, MRI, Langendorff

\section{INTRODUCTION}

Although Huntington's disease (HD) is unequivocally a neurological disease, the HD gene is ubiquitously expressed, and the protein is found in most peripheral tissues including skeletal muscle, liver and heart [1]. Inclusions appear in all of these tissues, with number of inclusions increasing as the course of the disease progresses [1]. However, the functional

*Correspondence to: Prof. A. Jenny Morton, Department of Pharmacology, University of Cambridge, Tennis Court Road, Cambridge CB2 1PD, UK. Tel.: +44 1223 334057; E-mail: ajm41@cam.ac.uk. consequence of pathology in peripheral tissues remains completely unknown.

There is a growing body of evidence, both direct and circumstantial, for cardiac dysfunction in HD. For instance, cardiac failure is a major cause of death in HD patients [2-5]. In pre-manifest and early HD patients, subtle abnormalities of autonomic control of the cardiovascular system have been reported $[6,7]$. These progress in range and magnitude as the disease advances [7, 8]. Furthermore, it has been shown that there is dysfunctional cardiac circadian control in HD mouse models $[9,10]$. Collectively, the data suggest that cardiac abnormalities represent peripheral 
manifestations of the HD phenotype that may have significant implications for both morbidity and mortality. Cognitive function is progressively impaired in HD. However, despite the emerging link between HD and cardiovascular dysfunction in humans, investigation of cardiac function in animal models of HD has been comparatively neglected. Only one comprehensive study has been performed using HD mice, in which the authors reported evidence for severe cardiac failure in R6/2 mice at end-stage of disease [11]. Since chronic heart failure is an important risk factor for cognitive impairment [12-14], we hypothesize that progressive cardiac failure may potentiate the inevitable cognitive dysfunction in HD patients.

Here, we investigated cardiac function in R6/2 mice at mid-symptomatic stages of disease. We used R6/2 mice with a CAG repeat of 250 that typically live to around 24 weeks of age. We used MRI to visualize the functional consequences of cardiac abnormalities in R6/2 HD mice and to determine heart rate (HR), end diastolic volume (EDV), end systolic volume (ESV), stroke volume (SV), cardiac output (CO) and ejection fraction (EF) at earlier stages of the disease progression. We also used the isolated Langendorff preparations of the R6/2 mouse heart for detailed assessment of systolic and diastolic function.

\section{MATERIALS AND METHODS}

All components of this study were carried out in accordance with the UK Animals (Scientific Procedures) Act, 1986, and with the approval of the University of Cambridge Ethical Review Panel.

\section{Animals}

Mice were taken from a colony of R6/2 transgenic mice [15] established in the Department of Pharmacology, University of Cambridge, and maintained by backcrossing onto CBA $\times$ C57BL6N F1 female mice. Genotyping and $\mathrm{CAG}$ repeat length measurement were carried out by Laragen (Los Angeles, CA, USA) as described previously [16]. The transgenic mice used in this study had a mean CAG repeat length of $242 \pm 1$ (range 237-251). Mice were kept in home cages comprising single sex, single genotype groups of five (MRI mice) or 10 (Langendorff preparation mice). All of the mice lived in an enhanced environment with increased amounts of bedding and nesting materials. Clean cages were provided twice weekly with grade $8 / 10$-corncob bedding, and finely shredded paper for nesting. The mice were maintained on a 12:12 hour light/dark (LD) cycle, at a temperature of $21-23^{\circ} \mathrm{C}$ and a humidity of $55 \pm 10 \%$. The mice had ad libitum access to water (using water bottles with elongated spouts) and dry laboratory food (RM3(E) rodent pellets, Special Diet Services, Witham, UK). In addition, once a day, a mash was prepared by soaking $100 \mathrm{~g}$ dry food in $230 \mathrm{ml}$ water until the pellets were soft and fully expanded. The mash was placed on the cage floor, improving access to food and water for the R6/2 transgenic mice. This feeding regime has been shown previously to be beneficial [17].

\section{Magnetic Resonance Imaging (MRI)}

Ten R6/2 mice ( 5 male, 5 female) and 10 littermate WT mice ( 5 male, 5 female) were scanned at 13.2 weeks (range 12.9-13.7 weeks) and 16.5 weeks (range 16.1-16.9 weeks). One R6/2 mouse died in the interim period, so this mouse was excluded from all subsequent analyses.

Anaesthesia was induced and maintained with gaseous isoflurane $\left(1.5-2 \%\right.$ in $\left.11 / \mathrm{min} \mathrm{O}_{2}\right)$. A pressure sensor for respiration rate was used to monitor anaesthesia depth and adjust isoflurane rate to maintain consistency between animals (in the range 20-35 breaths per minute). Gating of the MRI sequences was achieved prospectively with ECG recording. In order to minimize scan time, we kept respiration and heart rates constant for each animal by altering anaesthesia levels. Since heart rates were artificially manipulated, they are not presented in the Results. Body temperature was measured with a rectal thermometer and a flowingwater heating blanket was used to regulate temperature throughout the experiment.

Imaging was performed at 4.7 Tesla with a Bruker BioSpec 47/40 system (Bruker Inc. Ettlingen, Germany). A birdcage coil of $12 \mathrm{~cm}$ was used for signal excitation and animals were positioned prone over a $2 \mathrm{~cm}$ surface coil for signal reception. After initial localization images, a 4-chamber view was acquired (FLASH, TR/TE $8 \mathrm{~ms} / 2.8 \mathrm{~ms}, 20-25$ frames, $2.56 \mathrm{~cm}$ FOV, 256 matrix, $1.5 \mathrm{~mm}$ slice thickness, bandwidth $70 \mathrm{kHz}$, flip angle $30^{\circ}$, NEX 8). Using this scan as a reference, short axis slices were arranged perpendicular to the septum to cover the left ventricle (LV). To improve contrast between the blood and the myocardium, saturation slices were placed over the pulmonary veins/left atrium for a black-blood sequence (FLASH, TR/TE $10.9 \mathrm{~ms} / 2.8 \mathrm{~ms}, 15-18$ frames, $2.56 \mathrm{~cm}$ FOV, 256 matrix, $1.1 \mathrm{~mm}$ slice thickness, bandwidth $70 \mathrm{kHz}$, flip angle $20^{\circ}$, NEX 6). Full LV coverage was achieved with no slice gap with 6-8 slices. Depending on the 
heart rate of the animal, total scan time ranged from 35 to 135 minutes.

\section{MRI cardiac measures}

Delineations of the left ventricle (LV) were made at each phase of the cardiac cycle, including the papillary muscles throughout. The regions from each slice were combined using Simpson's rule to provide end systolic volume (ESV) and end diastolic volume (EDV) using Segment v1.9 [18]. Systolic volume (SV) is the difference between these, and the ejection fraction (EF) is the ratio of SV to EDV. Cardiac output (CO) was calculated as the product of mean HR throughout the MRI examination with SV. All hearts were analysed by a single reviewer blind to genotype. To assess intrareviewer repeatability, hearts ( $3 \mathrm{WT}$ and $3 \mathrm{R} 6 / 2$ ) were randomly selected for repeated segmentation of the LV.

\section{MRI assessment of cardiac shape and motion}

In viewing the MRI cine frames as a movie, we observed that the R6/2 hearts appeared to have a different shape during contraction, in particular, that there was some bending of the LV.

To assess this, we conceived a simple measure of 'bending' of the left ventricle. We fitted three lines to the centroids of delineated LV blood pool regions. The first line was the best fit to the centroids of each slice to determine the 'midpoint' of the LV. Two further lines were found as the best fits to the slices above and below this midpoint respectively. The metric we considered is the angle made between these lines. An increase in the angle between lines of best fit in the upper and lower parts of the left ventricle indicates cardiac distortion. All hearts were analyzed by a single reviewer blind to genotype.

\section{Isolated perfused heart (Langendorff) preparations}

Seven male R6/2 mice and seven male littermate WT mice ( 16 weeks of age) were killed by $\mathrm{CO}_{2}$ inhalation and posterior cervical dislocation. After recording of body weight and crown rump length (CRL), a thoracotomy was performed and the heart was rapidly excised and immediately placed into ice-cold Krebs-Henseleit bicarbonate buffer. The heart was then cannulated via the aorta and perfused through the coronary arteries whilst lung, mediastinal, and peri-cardiac brown adipose tissue were excised. A pulmonary arteriotomy was also performed. Hearts were perfused at a constant pressure of $75 \mathrm{mmHg}$ [19]. A small flexible non-elastic balloon was inserted into the left ventricle through the left atrium. The balloon was filled with deionized water and attached to a rigid deionized water-filled catheter connected to a calibrated pressure transducer (Argon Medical Devices, Texas, USA). The volume of the balloon was adjusted by injection of deionized water with a $100 \mu \mathrm{L}$ 'Hamilton' syringe to obtain recordings of left ventricular end diastolic pressure between $5-10 \mathrm{mmHg}$ during the initial $15 \mathrm{~min}$ of stabilization, after which it was not adjusted further [19]. A re-circulating Krebs-Henseleit bicarbonate buffer solution containing (mM.L $\left.{ }^{-1}\right) 120 \mathrm{NaCl}, 4.7 \mathrm{KCl}, 1.2$ $\mathrm{MgSO}_{2} .7 \mathrm{H}_{2} \mathrm{O}, 1.2 \mathrm{KH}_{2} \mathrm{PO}_{4}, 25 \mathrm{NaHCO}_{3}, 10$ glucose, and $1.3 \mathrm{CaCl}_{2} \cdot 2 \mathrm{H}_{2} \mathrm{O}$ was filtered through a $5 \mu \mathrm{m}$ cellulose nitrate filter (Millipore, Bedford, MA, USA) and gassed with $\mathrm{O}_{2}: \mathrm{CO}_{2}(95: 5)$ at $37^{\circ} \mathrm{C}$. After the initial stabilisation period of 15 minutes, basal heart rate (HR), basal left ventricular systolic pressure (LVSP) and basal LVEDP were recorded. Basal left ventricular developed pressure (LVDP) was calculated as LVSPLVEDP. The maximum and minimum first derivatives of the left ventricular pressure $\left(\mathrm{dP} / \mathrm{d} t_{\max }\right.$ and $\left.\mathrm{dP} / \mathrm{d} t_{\min }\right)$ were calculated automatically using an M-PAQ data acquisition system (Maastricht Programmable AcQuisition System, Netherlands). Basal coronary flow rate (CFR) was calculated by timed collections of perfusate effluent volume from the pulmonary incision [20].

\section{Statistical analysis}

Data are expressed as mean \pm S.E.M. Langendorff data were compared statistically by the Student's $t$ test for unpaired data. MRI data were analyzed by repeated measures ANOVA, except where specifically mentioned in the Results. Statistical significance was accepted when $p<0.05$. Analyses were conducted using SigmaStat 3.5 (SPSS Inc., Chicago, USA), or MATLAB (MathWorks, Natick, MA, USA).

\section{RESULTS}

\section{Biometry}

Relative to WT mice, 16 week old R6/2 mice had significantly lower body weight and ponderal index (body weight/crown rump length ${ }^{3}$ ), but the crown rump length was unchanged (Table 1). R6/2 mice also had significantly lighter hearts, whether expressed as the absolute weight of the whole organ, or as the left and 
Table 1

Body and cardiac parameters derived from 16 week old mice used for Langendorff preparations

\begin{tabular}{lcc}
\hline & WT $(n=7)$ & R6/2 $(n=7)$ \\
\hline Body weight $(\mathrm{g})$ & $30.67 \pm 0.63$ & $23.58 \pm 0.91^{*}$ \\
Crown-rump length $(\mathrm{cm})$ & $7.35 \pm 0.09$ & $7.11 \pm 0.15$ \\
Ponderal index $\left(\mathrm{kg} / \mathrm{m}^{3}\right)$ & $77.26 \pm 2.59$ & $66.29 \pm 3.70^{*}$ \\
Heart weight & & \\
$\quad$ Whole, absolute $(\mathrm{g})$ & $0.14 \pm 0.01$ & $0.10 \pm 0.01^{*}$ \\
Whole, relative $(\%$ total $)$ & $0.47 \pm 0.02$ & $0.45 \pm 0.02$ \\
LV+ septum, absolute $(\mathrm{g})$ & $0.10 \pm 0.003$ & $0.07 \pm 0.004^{*}$ \\
LV+ septum, relative $(\%$ total $)$ & $69.79 \pm 1.96$ & $71.08 \pm 2.27$ \\
RV, absolute $(\mathrm{g})$ & $0.03 \pm 0.005$ & $0.02 \pm 0.002^{*}$ \\
$\quad$ RV, relative $(\%$ total $)$ & $20.37 \pm 2.87$ & $19.03 \pm 2.18$ \\
Heart rate (beats/min) & $338.0 \pm 6.20$ & $318.00 \pm 10.80$ \\
Coronary flow rate $(\mathrm{ml} / \mathrm{min})$ & $2.21 \pm 0.13$ & $1.45 \pm 0.18^{*}$ \\
\hline
\end{tabular}

$\mathrm{LV}$, left ventricle; RV, right ventricle. Data are means \pm SEM. Significant differences are $\mathrm{R} 6 / 2$ compared to WT mice. ${ }^{*} p<0.05$.

right compartments (Table 1). However, the change in heart mass relative to body weight was not significantly different between genotypes (Table 1).

\section{Langendorff preparation}

When compared to WT mice, 16 week old R6/2 isolated mouse hearts showed lower left ventricular developed pressure (LVDP), impaired myocardial contractility $\left(\mathrm{dP} / \mathrm{dt}_{\max }\right)$ and relaxability $\left(\mathrm{dP} / \mathrm{dt}_{\min } ;\right.$ Fig. 1$)$, with reduced coronary flow rate but no change in basal heart rate (Table 1). There was a trend for left ventricular end diastolic pressure (LVEDP) to be increased
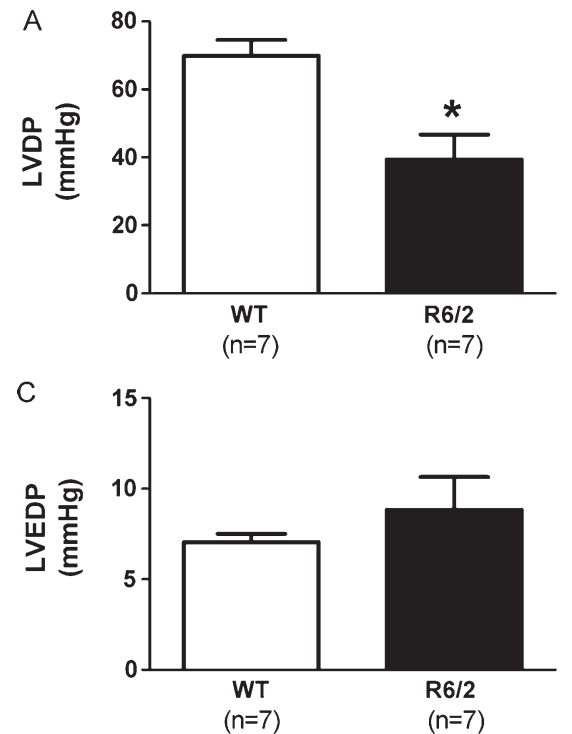

in R6/2 mice compared to WT mice, but this did not reach statistical significance (Fig. 1).

\section{Magnetic resonance imaging}

At 13 weeks of age, end diastolic volume (EDV) and end systolic volume (ESV) were reduced by $17 \pm 4 \%$ $(p<0.002)$ and $28 \pm 9 \%(p<0.02)$ respectively, in R6/2 compared with WT mice (Table 2). By 16 weeks, abnormalities increased to $37 \pm 7 \%(p<0.00001)$ and $53 \pm 11 \%(p<0.00001)$, respectively (Table 2$)$. A paired Student's $t$-test of values for EDV $(p<0.002)$ and ESV $(p<0.04)$ between 13 and 16 weeks confirmed that the genotype difference was progressive. Measures of stroke volume $(\mathrm{SV}, 9 \pm 5 \%, p=0.24)$ and cardiac output $(\mathrm{CO}, 11 \pm 7 \%, p=0.22)$ at 13 weeks of age revealed non-significant reductions in $\mathrm{R} 6 / 2$ compared with WT mice (Table 2). Ejection fraction showed a non-significant increase at 13 weeks (EF, $10 \pm 5 \%, p=0.19)$. By 16 weeks of age however, SV and $\mathrm{CO}$ became significantly reduced in R6/2 compared with WT mice (SV, $26 \pm 6 \%, p<0.0001$; CO, $31 \pm 7 \%, p<0.0005$; Table 2), while EF was increased in $\mathrm{R} 6 / 2$ mice $(18 \pm 5 \%, p<0.005$; Table 2$)$.

Visual inspection of the MRI images revealed that R6/2 hearts were smaller than WT hearts (Fig. 2), and had marked visual differences in the cardiac cycle (Video S1). Video recordings of the beating hearts suggest that hearts from R6/2 mice have a distorted shape during contraction relative to WT mice.
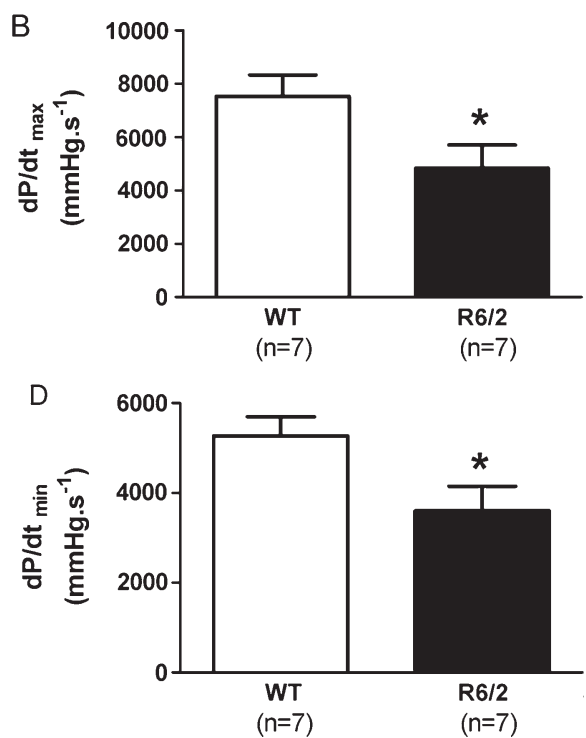

Fig. 1. Cardiac systolic and diastolic function measured from Langendorff preparations. Panel A shows left ventricular developed pressure (LVDP), and panel C left ventricular end diastolic pressure (LVEDP). $\mathrm{dP}_{\mathrm{d}} \mathrm{dt}_{\max }$ and $\mathrm{dP} / \mathrm{dt}_{\min }$ are shown in B and D. Data are means $\pm \mathrm{SEM}$. Significant differences are R6/2 compared to WT mice. ${ }^{*} p<0.05$. 
Table 2

Cardiac parameters derived from mice used for MRI scans

\begin{tabular}{|c|c|c|c|c|}
\hline & \multicolumn{2}{|c|}{ Scan 1 (13 weeks) } & \multicolumn{2}{|c|}{ Scan 2 (16 weeks) } \\
\hline & $\overline{\mathrm{WT}}(n=10)$ & $\mathrm{R} 6 / 2(n=9)$ & $\overline{\mathrm{WT}}(n=10)$ & $\mathrm{R} 6 / 2(n=9)$ \\
\hline Age (weeks) & $13.1 \pm 0.2$ & $13.3 \pm 0.1$ & $16.7 \pm 0.1$ & $16.4 \pm 0.1$ \\
\hline Body weight (g) & $27.7 \pm 1.0$ & $21.1 \pm 1.4^{* *}$ & $29.3 \pm 1.3$ & $19.0 \pm 1.1^{* * *}$ \\
\hline $\mathrm{EDV}(\mu \mathrm{l})$ & $45.3 \pm 1.5$ & $37.3 \pm 1.6^{* *}$ & $46.0 \pm 1.4$ & $29.1 \pm 1.5^{* * *}$ \\
\hline $\operatorname{ESV}(\mu \mathrm{l})$ & $20.9 \pm 1.7$ & $15.0 \pm 1.4^{*}$ & $18.2 \pm 1.4$ & $8.6 \pm 1.1^{\text {****}}$ \\
\hline $\mathrm{SV}(\mu \mathrm{l})$ & $24.4 \pm 1.0$ & $22.3 \pm 1.4$ & $27.8 \pm 1.2$ & $20.6 \pm 0.7^{* * *}$ \\
\hline $\mathrm{CO}(\mathrm{ml} / \mathrm{min})$ & $6.9 \pm 0.5$ & $6.1 \pm 0.4$ & $8.1 \pm 0.5$ & $5.5 \pm 0.5^{* * *}$ \\
\hline $\mathrm{EF}(\%)$ & $54.0 \pm 3.0$ & $60.0 \pm 3.0$ & $61.0 \pm 2.0$ & $71.0 \pm 2.0^{* *}$ \\
\hline LV bending angle $\left({ }^{\circ}\right)$ & $13.3 \pm 1.5$ & $14.3 \pm 1.5$ & $11.0 \pm 1.2$ & $15.0 \pm 1.4^{*}$ \\
\hline
\end{tabular}

$\mathrm{EDV}$, end diastolic volume; ESV, end systolic volume; SV, stroke volume; $\mathrm{CO}$, cardiac output; $\mathrm{EF}$, ejection fraction; $\mathrm{LV}$, left ventricle. Data are means \pm SEM. Significant differences are R6/2 compared to WT mice. ${ }^{*} p<0.05$, ${ }^{* *} p<0.01,{ }^{* * *} p<0.001$.
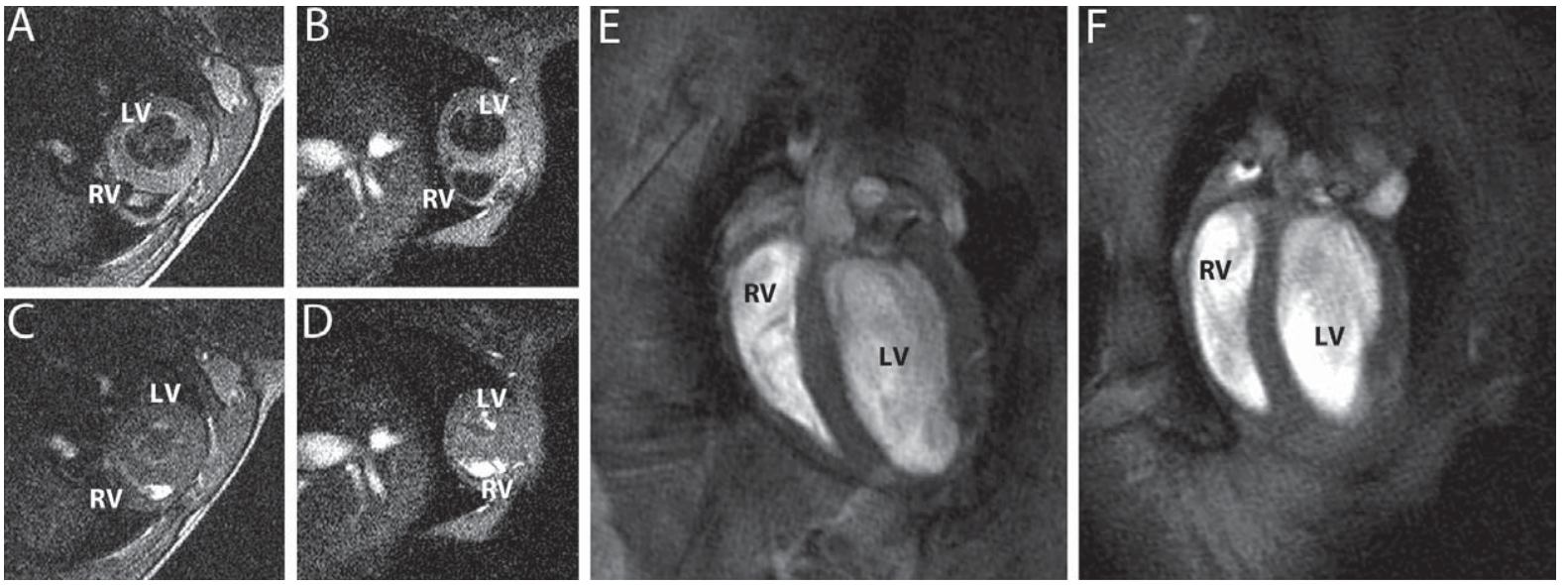

Fig. 2. Short axis views of 16 week old mouse hearts in diastole (A, WT; B, R6/2) and systole (C, WT; D, R6/2), showing the reduced size of the R6/2 mouse heart. This difference in size is more obvious in the four chamber views (E, WT; F, R6/2).

A
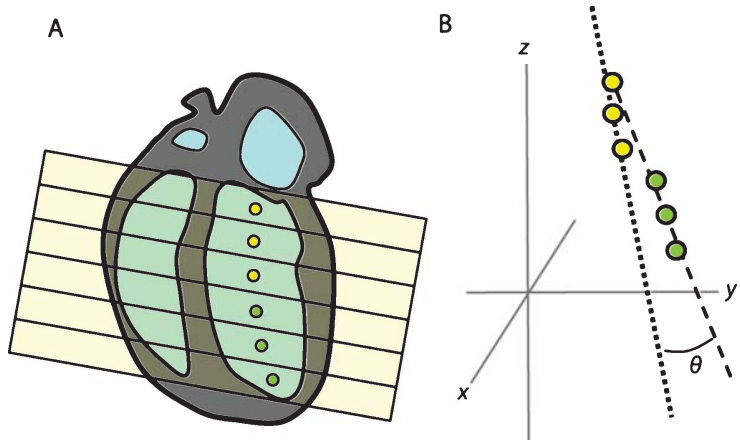

Fig. 3. Calculation of LV bending angle. The LV endocardium is delineated on short axis slices to calculate the centroid of each slice, shown in the 4-chamber view (A). A 3D line of best fit to these points is calculated for the upper and lower part of the LV chamber. (B). The angle $\theta$ between these lines gives a simple parameter that describes the 'shape' of the LV.

Measurement of LV bending (a quantitative measure of cardiac distortion; Fig. 3) revealed no difference between genotypes at 13 weeks, but by 16 weeks the bending angle in R6/2 mice was significantly greater than in WT mice $(p<0.05$, Table 2$)$. An additional finding, and one that requires further investigation, is the appearance of 'adhesions' of the apex through the pericardium in the images from all 16 week old R6/2 mice (Fig. 4). These phenomena were not seen in 13 week old R6/2 or WT mice at either age.

Repeated measurements of EDV were within $7 \%$ (mean absolute difference). For comparison, the standard deviation of EDV amongst WT mice is 10\%, and the difference between groups at the second time point in EDV is $40 \%$ of the WT value so variability in drawing of regions cannot explain the differences found.

\section{DISCUSSION}

In vivo MRI and ex vivo isolated Langendorff investigation of R6/2 mice hearts showed that compared to WT mice hearts they have significant differences in 


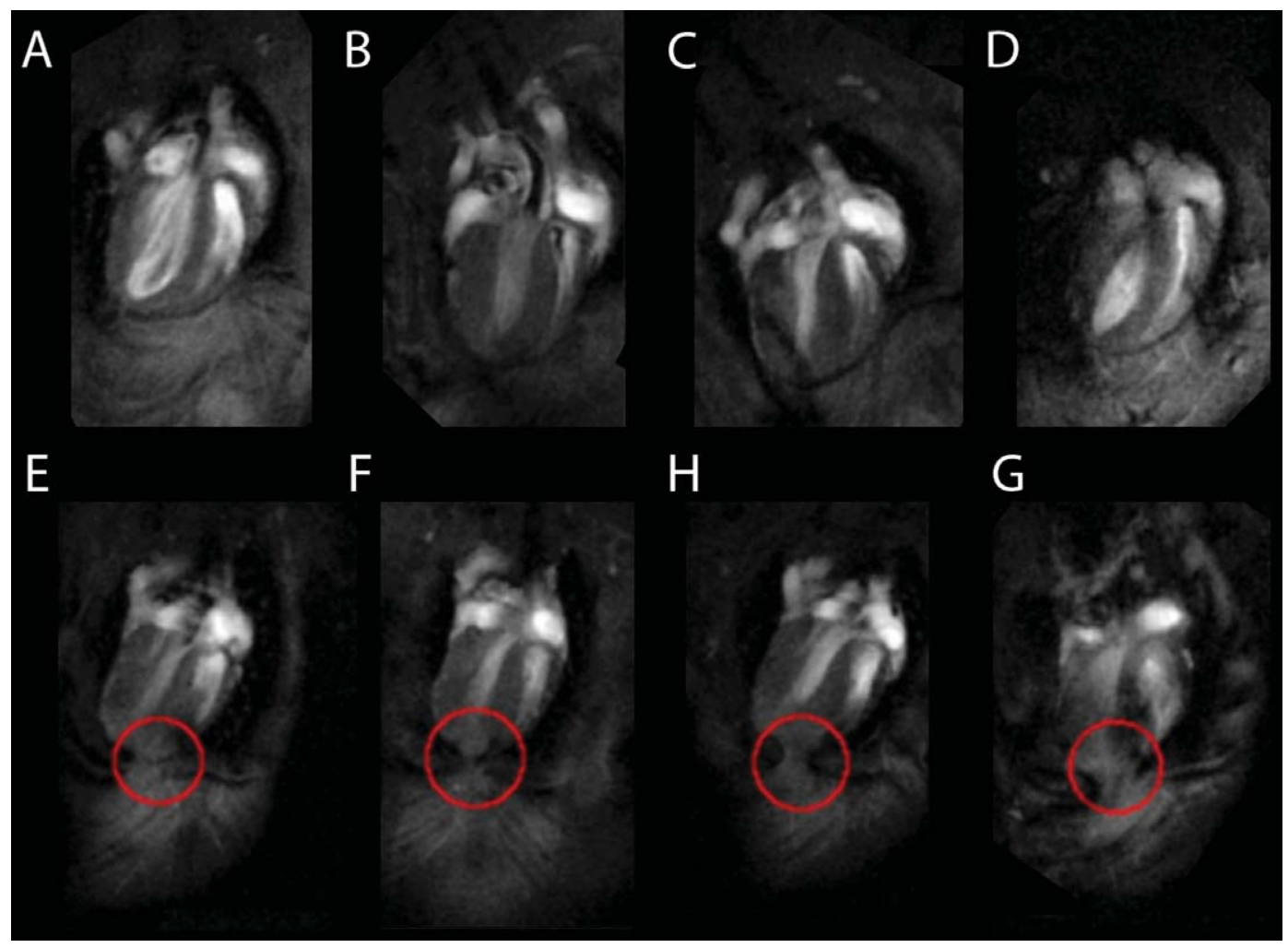

Fig. 4. MR images from individual WT mice (A-D), and R6/2 mice (E, G) at 16 weeks of age. R6/2 images show apparent adhesions of the apex of the heart to the pericardium (circles). These adhesions were not seen in the hearts of WT mice (A-D).

both morphology and function, and that these differences increase in severity as HD progresses. Data from both the MRI and the Langendorff isolated heart preparation show that $\mathrm{R} 6 / 2$ mice are less able to generate left ventricular pressure, with significant impairment of myocardial contractility and relaxability. The cardiac phenotype is typical of weak and stiff hearts $[19$, 21]. R6/2 mice also show a significantly lower coronary flow rate, indicating reduced coronary reserve and thereby increased susceptibility to ischaemic heart injury [22].

The reduced body weight and decreased ponderal index of R6/2 mice relative to WT controls confirms that HD mice are disproportionally thin for their length. This is consistent with the adult-onset loss of body weight and atrophy of skeletal muscle that has been well described $[23,24]$. In contrast, the in vivo and $e x$ vivo cardiac morphology data suggest that the reduction in size of the hearts of $\mathrm{R} 6 / 2$ mice is proportional to the overall reduction in body weight. The phenotype in R6/2 mice develops quickly, and mice with $\sim 250$ CAG repeats typically die between 20-24 weeks of age [25]. However, obvious physical indications of the phenotype, other than a failure to gain weight at the same rate as WT mice, are largely absent before 12 weeks of age. It is interesting, therefore, that we could detect cardiac abnormalities (reductions in EDV and ESV) by 13 weeks. We did not measure heart weights in 13 week old mice, but as their body weights were already significantly lower than WT mice, it is reasonable to assume that some cardiac atrophy may be present at this age. The much greater reductions in cardiac parameters (EDV, ESV, SV, CO and EF) in 16 week old R6/2 mice measured from in vivo MRI scans is in accordance with the overall reduction in the size of the heart in these mice, and correlates well with data from the Langendorff preparation. Although a number of our observations may be directly related to heart size, data from the perfused ex vivo hearts (reduced LVDP, impaired myocardial contractility and relaxability, and reduced coronary flow rate) demonstrate that there is also an abnormality of cardiac function. These deficits, combined with the mechanical distortions and adhesions that we detected in MRI scans, suggest that cardiac dysfunction is an important part of the R6/2 phenotype. 
The in vivo MRI data revealed a greater bending angle of the left ventricle by 16 weeks of age in R6/2 relative to WT mice. This suggests greater cardiac distortion in HD hearts compared to controls. The significance of this observation is not known, but the reduction in efficiency of the heart beat and the presence of stress markers indicate that it is likely to be deleterious. The heart is enclosed by the pericardium that is attached to the posterior surface of the sternum by sternopericardial ligaments. In R6/2 mice, the heart develops normally, with atrophy beginning only after the animals are around 13 weeks old. The normal crown-rump length of 16 week old R6/2 mice suggests that the atrophied heart is attached to a non-atrophied ribcage. The stress markers were not seen in 13 week old R6/2 or WT mice of either age, so it is possible that they are related to the development of skeletal abnormalities such as lordokyphosis as the disease progresses in R6/2 mice [24]. This may be the cause of the change in LV bending angle that we have observed. We suggest that this is likely to contribute to the inefficiency of the HD mouse heart function as the disease progresses.

Cardiac failure is a major cause of death in HD patients [2-5]. Here we have demonstrated in vivo cardiac dysfunction in the R6/2 mouse model of HD. These data also add to the body of work demonstrating the usefulness of the R6/2 mouse as a model of HD. We have not attempted to measure cognitive function in this study. However, there is a strong correlation between cardiac failure and cognitive impairment in humans [12-14]. Furthermore, we have previously demonstrated progressive cognitive decline in R6/2 mice [26-30]. Thus we hypothesize that chronic heart failure in R6/2 mice may contribute not only to the deterioration in physical condition, but also to the cognitive dysfunction that is a feature of HD. We suggest that heart function in mid to late stage HD patients is worthy of further study.

\section{ACKNOWLEDGMENTS}

This work was funded by a grant from CHDI Foundation Inc. (http://www.highqfoundation.org/) to AJM. We thank Karen Skelton for technical assistance.

\section{REFERENCES}

[1] Sathasivam K, Hobbs C, Turmaine M, Mangiarini L, Mahal A, Bertaux F, et al. Formation of polyglutamine inclusions in non-CNS tissue. Hum Mol Genet. 1999;8(5):813-22.
[2] Chiu E, Alexander L. Causes of death in Huntington's disease. Med J Aust. 1982;1(4):153.

[3] Lanska DJ, Lanska MJ, Lavine L, Schoenberg BS. Conditions associated with Huntington's disease at death. A case-control study. Arch Neurol. 1988;45(8):878-80.

[4] Lanska DJ, Lavine L, Lanska MJ, Schoenberg BS. Huntington's disease mortality in the United States. Neurology. 1988;38(5):769-72.

[5] Sorensen SA, Fenger K. Causes of death in patients with Huntington's disease and in unaffected first degree relatives. J Med Genet. 1992;29(12):911-4.

[6] Aziz NA, Anguelova GV, Marinus J, van Dijk JG, Roos RA. Autonomic symptoms in patients and pre-manifest mutation carriers of Huntington's disease. Eur J Neurol. 2010;17(8):1068-74.

[7] Kobal J, Melik Z, Cankar K, Bajrovic FF, Meglic B, Peterlin B, et al. Autonomic dysfunction in presymptomatic and early symptomatic Huntington's disease. Acta Neurol Scand. 2010;121(6):392-9.

[8] Andrich J, Schmitz T, Saft C, Postert T, Kraus P, Epplen JT, et al. Autonomic nervous system function in Huntington's disease. J Neurol Neurosurg Psychiatry. 2002;72(6):726-31.

[9] Maywood ES, Fraenkel E, McAllister CJ, Wood N, Reddy $\mathrm{AB}$, Hastings $\mathrm{MH}$, et al. Disruption of peripheral circadian timekeeping in a mouse model of Huntington's disease and its restoration by temporally scheduled feeding. J Neurosci. 2010;30(30):10199-204.

[10] Kudo T, Schroeder A, Loh DH, Kuljis D, Jordan MC, Roos $\mathrm{KP}$, et al. Dysfunctions in circadian behavior and physiology in mouse models of Huntington's disease. Exp Neurol. 2011;228(1):80-90.

[11] Mihm MJ, Amann DM, Schanbacher BL, Altschuld RA, Bauer JA, Hoyt KR. Cardiac dysfunction in the R6/2 mouse model of Huntington's disease. Neurobiol Dis. 2007;25(2):297-308

[12] Pressler SJ. Cognitive functioning and chronic heart failure: A review of the literature (2002-July 2007). J Cardiovasc Nurs. 2008;23(3):239-49.

[13] Cameron J, Ski CF, Thompson DR. Cognitive impairment in chronic heart failure and the need for screening. Am J Cardiol. 2011;107(10):1547-8.

[14] Harkness K, Demers C, Heckman GA, McKelvie RS. Screening for cognitive deficits using the Montreal cognitive assessment tool in outpatients $\geq 65$ years of age with heart failure. Am J Cardiol. 2011;107(8):1203-7.

[15] Mangiarini L, Sathasivam K, Seller M, Cozens B, Harper A, Hetherington $\mathrm{C}$, et al. Exon 1 of the HD gene with an expanded CAG repeat is sufficient to cause a progressive neurological phenotype in transgenic mice. Cell. 1996;87:493-506.

[16] Duzdevich D, Li J, Whang J, Takahashi H, Takeyasu K, Dryden DT, et al. Unusual structures are present in DNA fragments containing super-long Huntingtin CAG repeats. PLoS One. 2011;6(2):e17119.

[17] Carter RJ, Hunt MJ, Morton AJ. Environmental stimulation increases survival in mice transgenic for exon 1 of the Huntington's disease gene. Mov Dis. 2000;15(5):925-37.

[18] Heiberg E, Ugander M, Engblom H, Götberg M, Olivecrona GK, Erlinge D, et al. Automated quantification of myocardial infarction from MR images by accounting for partial volume effects: Animal, phantom, and human study. Radiology. 2008;246(2):581-8.

[19] Sutherland FJ, Shattock MJ, Baker KE, Hearse DJ. Mouse isolated perfused heart: Characteristics and cautions. Clin Exp Pharm Phys. 2003;30:867-78. 
[20] Niu YG, Evans RD. Myocardial metabolism of triacylglycerol-rich lipoproteins in type 2 diabetes. J Physiol. 2009;587(13):3301-15.

[21] Reichelt ME, Willems L, Hack BA, Peart JN, Headrick JP. Cardiac and coronary function in the Langendorff-perfused mouse heart model. Exp Physiol. 2008;94(1):54-70.

[22] Hauton D, Ousley V. Prenatal hypoxia induces increased cardiac contractility on a background of decreased capillary density. BMC Cardiovasc Disorders. 2009;9:1.

[23] Carter RJ, Lione LA, Humby T, Mangiarini L, Mahal A, Bates GP, et al. Characterization of progressive motor deficits in mice transgenic for the human Huntington's disease mutation. J Neurosci. 1999; 19(8):3248-57.

[24] Ribchester RR, Thomson D, Wood NI, Hinks T, Gillingwater TH, Wishart TM, et al. Progressive abnormalities in skeletal muscle and neuromuscular junctions of transgenic mice expressing the Huntington's disease mutation. Eur J Neurosci. 2004;20(11):3092-114

[25] Wood NI, Carta V, Milde S, Skillings EA, McAllister CJ, Ang $\mathrm{YL}$, et al. Responses to environmental enrichment differ with sex and genotype in a transgenic mouse model of Huntington's disease. PLoS One. 2010;5(2):e9077.
[26] Lione LA, Carter RJ, Hunt MJ, Bates GP, Morton AJ, Dunnett SB. Selective discrimination learning impairments in mice expressing the human Huntington's disease mutation. J Neurosci. 1999;19(23):10428-37.

[27] Morton AJ, Hunt MJ, Hodges AK, Lewis PD, Redfern AJ, Dunnett SB, et al. A combination drug therapy improves cognition and reverses gene expression changes in a mouse model of Huntington's disease. Eur J Neurosci. 2005;21(4):855-70.

[28] Pallier PN, Maywood ES, Zheng Z, Chesham JE, Inyushkin AN, Dyball R, et al. Pharmacological imposition of sleep slows cognitive decline and reverses dysregulation of circadian gene expression in a transgenic mouse model of Huntington's disease. J Neurosci. 2007;27(29):7869-78.

[29] Pallier PN, Morton AJ. Management of sleep/wake cycles improves cognitive function in a transgenic mouse model of Huntington's disease. Brain Res. 2009;1279:90-8.

[30] Wood NI, Glynn D, Morton AJ. "Brain training" improves cognitive performance and survival in a transgenic mouse model of Huntington's disease. Neurobiol Dis. 2011;42(3):427-37 